\title{
APORTACIONES AL CONOCIMIENTO DE LA DIETA DE CERROPHIDION PETLALCALENSIS (MARCO ANTONIO LÓPEZ LUNA, RICHARD C. VOGT, MIGUEL ÁNGEL DE LA TORRE LORANCA, 1999)(VIPERIDAE).
}

\section{CONTRIBUTIONS TO THE KNOWLEDGE OF THE DIET OF CERROPHIDION PETLALCALENSIS (MARCO ANTONIO LÓPEZ LUNA, RICHARD C. VOGT, MIGUEL ÁNGEL DE LA TORRE LORANCA, 1999)(VIPERIDAE).}

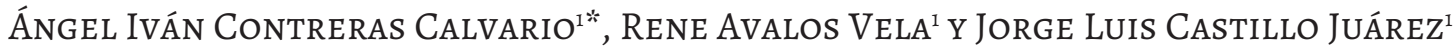 \\ ${ }^{1}$ Universidad Veracruzana, Facultad de Ciencias Biológicas y Agropecuarias, camino viejo Peñuela-Amatlán de los Reyes. S/n. 94945. Mpio. Amatlán \\ de los Reyes, Veracruz, México. \\ "Correspondent: acontrerascalvario@gmail.com
}

Abstract.- Novel data about the consumption of reptiles as a part of the diet of Cerrophidion petlalcalensis is provided.

Keywords.- Feeding, Montane pitviper, Natural history, Petlalcala montane pitviper, Viper.

Resumen.- Se aportan datos novedosos acerca del consumo de reptiles como parte de la dieta de Cerrophidion petlalcalensis.

Palabras clave.- Alimentación, Historia natural, Nauyaca de frío, Nauyaca del cerro Petlalcala, Vipérido.

Cerrophidion petlalcalensis es una especie que anteriormente se reportaba únicamente en su localidad tipo en el cerro Petlalcala, municipio de San Andrés Tenejapan, Veracruz, aunque recientemente se han descubierto otras locaciones dentro de la Sierra de Zongolica, la cual forma parte de la Sierra Madre de Oaxaca en elevaciones entre los 1354 y 2500 msnm en Veracruz y Oaxaca (López-Luna et al., 1999; Schramer et al., 2018). De esta especie se sabe relativamente poco sobre su historia natural, y respecto a su dieta solo han identificado anfibios y mamíferos. Se conoce que se alimenta de salamandras de los géneros Aquiloeurycea, Isthmura, Pseudoeurycea y Thorius (López-Luna et al., 1999; Schramer et al., 2018), anuros del género Craugastor (Heimes, 2016; Schramer et al., 2018), y musarañas del género Cryptotis (López-Luna et al., 1999).

En la recién publicada síntesis de la dieta del género Cerrophidion se menciona que cuatro de las cinco especies del género mantienen una alimentación generalista, debido a la diversidad de presas que consumen, tales como artrópodos (ortópteros, arácnidos y chilópodos), anfibios (caudados y anuros), reptiles (escamados), así como pequeños mamíferos (roedores e insectívoros) y aves (paseriformes), e incluso se reporta canibalismo en C. godmani (Schramer et al., 2018).

Durante una excursión de campo en el cerro Petlalcala, Municipio de San Andrés Tenejapan, Veracruz, México (18 ${ }^{\circ} 46^{\prime} 40.009$ "N, 976'7.482"O) 2120 m.s.n.m. a las 13:22 horas, el 24 de noviembre de 2018, se observó una hembra adulta (Fig. 1) de la especie $C$. petlalcalensis (Longitud hocico-cloaca $=38.5 \mathrm{~cm}$, Longitud Total $=43.5 \mathrm{~cm}$ ), asoleándose inmóvil sobre el suelo del bosque. El individuo se contuvo en un saco para serpientes, (ITSZ-A-091; permiso de recolección SEMARNAT-08-049, Oficio Núm.SGPA / DGVS / 02924/15 emitido a Erasmo Cázares-Hernández y colaboradores) para posteriormente ser 


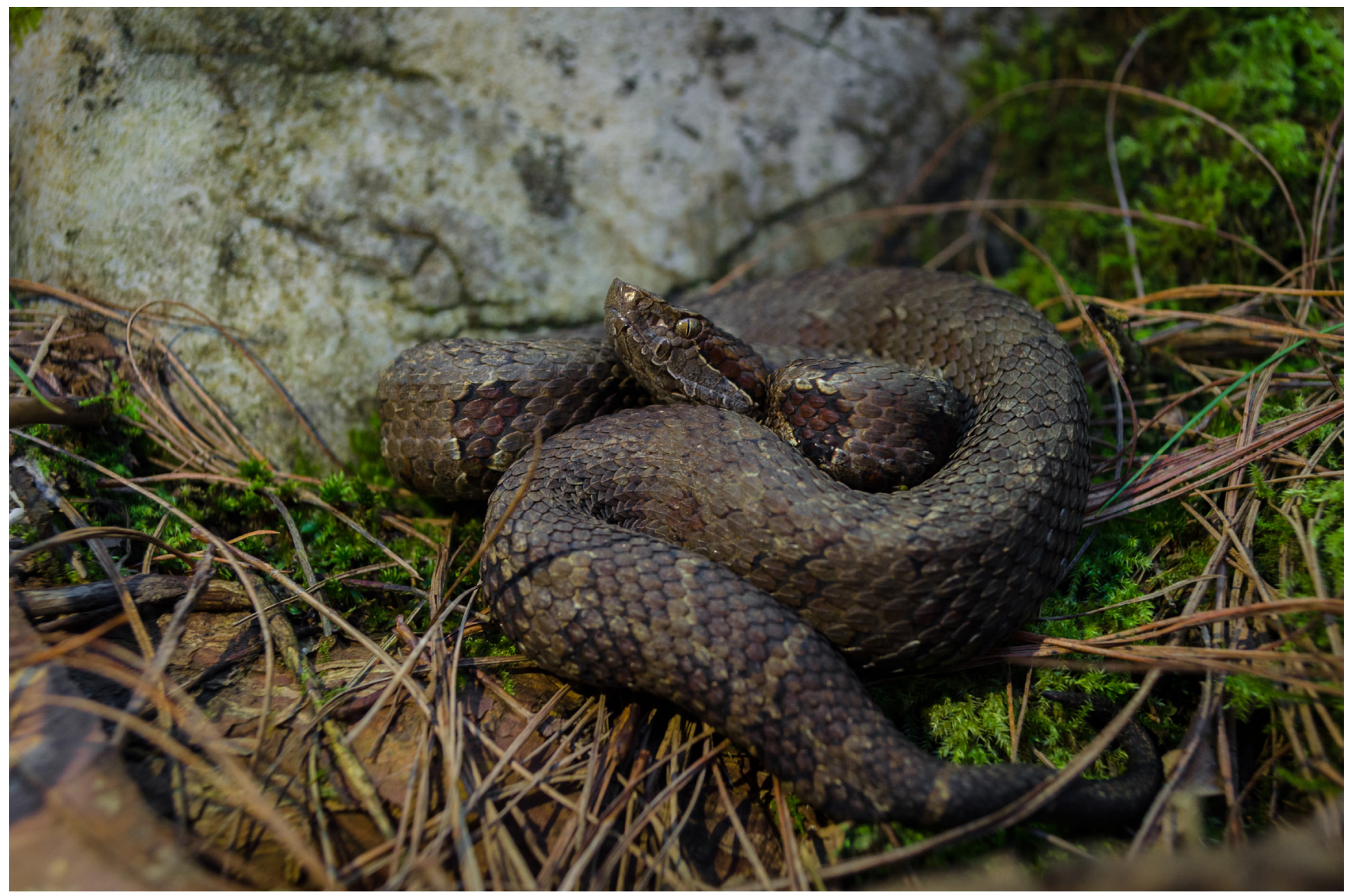

Figure 1. Female of Cerrophidion petlalcalensis from San Andres Tenejapan type locality. Photograph by Rene Avalos Vela.

Figura 1. Hembra de Cerrophidion petlalcalensis de su localidad tipo en San Andrés Tenejapan. Fotografía de Rene Avalos Vela.

fotografiado, el ejemplar defecó dentro del contenedor y las excretas fueron conservadas en formalina al 30\%, para poder analizar su contenido.

El ejemplar fue liberado el mismo día en la zona del avistamiento posterior a ser fotografiado y realizarle merística, de igual forma se tomaron los datos de latitud y longitud del sitio. Se utilizó un estereoscópico FORTY American Optical para realizar las observaciones de los objetos en las excretas.

En la muestra de excreta identificamos 26 escamas alargadas, que pertenecen a la región ventral de alguna especie de serpiente (Fig. 2A), así como escamas triangulares que podrían pertenecer a la misma presa (Fig. 2B).

Se obtuvieron además numerosas escamas (Fig. 2C), que por sus características determinamos que pertenecen a alguna especie del género Sceloporus, posiblemente $S$. formosus, $S$. mucronatus o $S$. variabilis ya que estas especies se distribuyen en dicha localidad de acuerdo con De la torre-Loranca (1999) y KellyHernández (2017).

De igual forma observamos restos óseos de aproximadamente $4 \mathrm{~mm}$ de longitud, así como un diente inoculador perteneciente a un vipérido (Fig. 2D).

Al estar poco estudiado su comportamiento y ser poca la información en vida silvestre aún se desconocen muchos aspectos de los hábitos alimenticios en esta especie. Otras especies del género Cerrophidion son generalistas en su dieta por lo tanto discutimos la posibilidad de que C. petlalcalensis también lo sea debido a las presas reportadas hasta el momento, e incluso el material fecal analizado sugiere la probabilidad de incluir vipéridos en su alimentación, pues esto ha sido reportado en otra especie de Cerrophidion (Schramer et al., 2018) y en otros miembros de la familia Viperidae (Mocino-Deloya et al., 
2009, Mendoza-Roldan \& Fernández-Lucero, 2011,). Tampoco descartamos que pudiera tratarse de una ingesta accidental del diente inoculador del mismo ejemplar. Las observaciones de historia natural y de las presas puntuales de esta especie son aún más escasas que las de algunos de sus congéneres por lo que necesitan ser documentadas.

En este trabajo reportamos por primera vez la ingesta de reptiles dentro de la dieta de $C$. petlalcalensis, consideramos relevante esta observación pues incrementa el conocimiento acerca de la historia natural de la especie.
Agradecimientos.- Agradecemos a Steven Bol por la colaboración en campo, Yaqueline A. Gheno por facilitarnos el estereoscopio, al Instituto Tecnológico Superior de Zongolica, Ramiro Sánchez-Uranga (Director General), Yamil VargasRivera (Director Adjunto de Investigación) y a Erasmo Cazares Hernández (colección científica ITSZ).

\section{LITERATURA CITADA}

De la Torre-Loranca, M.A. 1999. Anfibios y reptiles del cerro Petlalcala, municipio de San Andrés Tenejapan, Veracruz, México. Tesis de licenciatura. Facultad de Ciencias Biológicas y

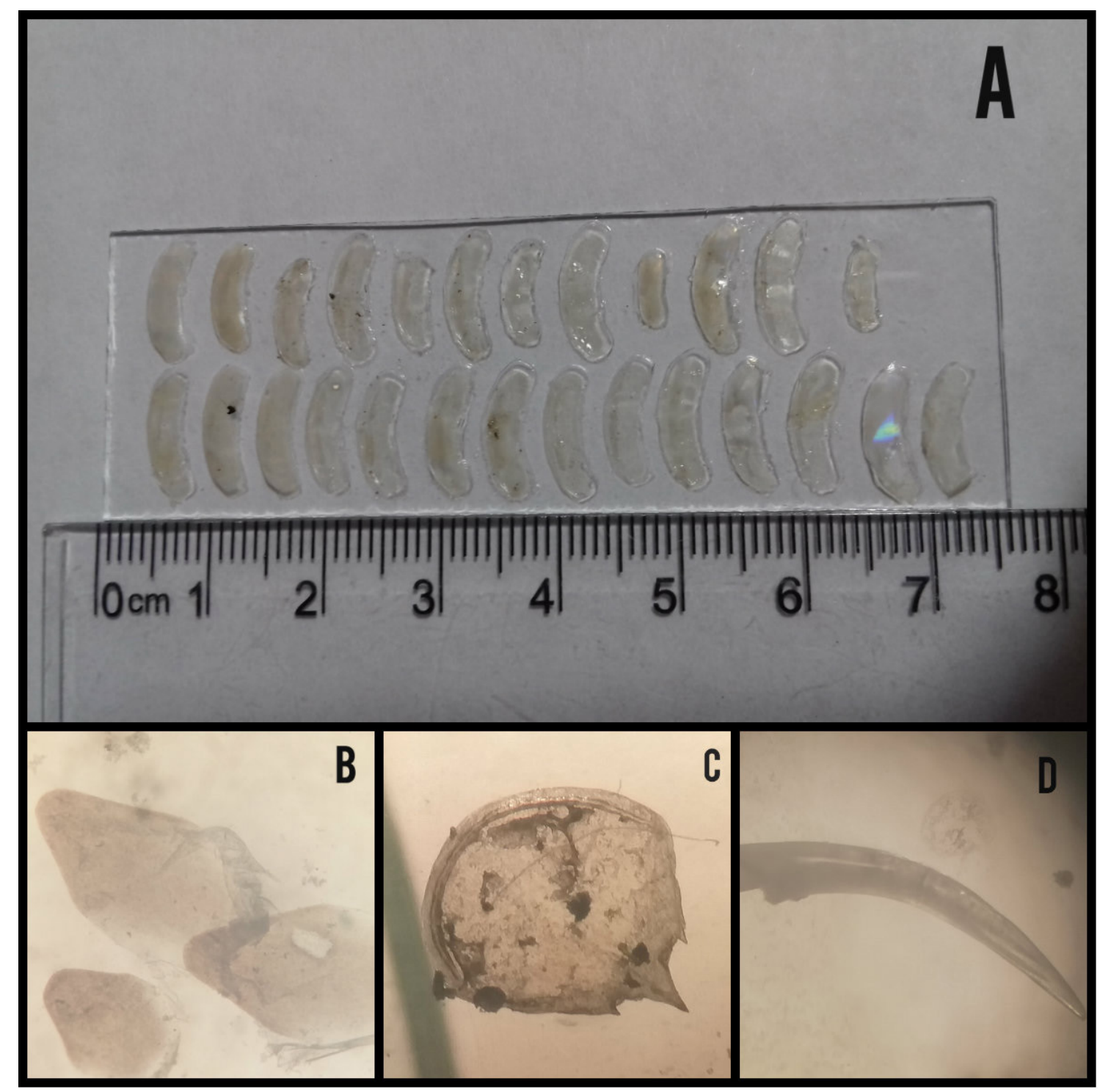

Figure 2. Samples of the content in the excreta. Ventral scales (A), triangular scales (B), mucronated scales (C), fang (D). Photograph by Jorge L. Castillo Juárez.

Figura 2. Muestras del contenido en la excreta. Escamas ventrales (A), escamas triangulares (B), escama mucronada (C), colmillo inoculador (D). Fotografía de Jorge L. Castillo Juárez. 
Agropecuarias, Universidad Veracruzana, Amatlán de los Reyes, Veracruz. México.

Heimes, P. 2016. Herpetofauna mexicana: Snakes of Mexico. Edition Chimaira. 411-412.

Kelly-Hernández, A. 2017. Actualización de la herpetofauna del cerro Petlalcala, municipio de San Andrés Tenejapan, Veracruz, México. Tesis de licenciatura. Facultad de Ciencias Biológicas y Agropecuarias, Universidad Veracruzana, Amatlán de los Reyes, Veracruz. México.

López-Luna, M.A., R. Vogt \& M.A. de la Torre-Loranca. 1999. A New Species of Montane Pitviper from Veracruz, México. Herpetologica 55(3):382-389.
Mendoza-Roldan, J.S. \& M. Fernández-Lucero. 2011. A prey item not previously recorded for Bothrops asper: a case of ophiophagy involving two sympatric pit viper species. Herpetotropicos: Tropical Amphibians \& Reptiles 5(2):107-110.

Mocino-Deloya, E., K. Setser, J.M. Pleguezuelos, A. Kardon \& D. Lazcano. 2009. Cannibalism of nonviable offspring by postparturient Mexican lance-headed rattlesnakes, Crotalus polystictus. Animal Behaviour 77(1):145-150.

Schramer, T.D., M.A. De La Torre-Loranca, M. Salazar-Saavedra, Y. Kalki \& D.B. Wylie. 2018. Additional diet information for Cerrophidion godmani (Günther, 1863) And a dietary synopsis of the genus Cerrophidion Campbell and Lamar, 1992. Revista Latinoamericana de Herpetología 1(2):35-43. 